\title{
An Overview of the Studies on Diastasis Recti Abdominis in Postpartum Women
}

\author{
Aswini $\mathrm{D}^{1}$ and Srihari Sharma KN ${ }^{2 *}$ \\ ${ }^{1}$ Dayananda Sagar University, India \\ ${ }^{2}$ Assistant Professor, Dayananda Sagar University, India \\ Submission: February 04, 2019 ; Published: April 18, 2019 \\ *Corresponding author: Srihari Sharma KN, Assistant Professor, Dayananda Sagar University, India
}

\begin{abstract}
Introduction: Local and systemic changes that occur during pregnancy and child birth return to pre-pregnancy state during the postpartum period which is marked by musculoskeletal issues due to hormonal influence. This in turn adversely affects the rectus abdominis muscle causing Diastasis Recti Abdominis (DRA).
\end{abstract}

Objectives: To analyze the prevalence, commonly used assessment techniques, treatment options, and research output of DRA in postpartum women.

Methods: Electronic search of Pub Med, Science Direct, Ovid, Scopus, and Web of Science was carried out. A data extraction form was structured and the analysis was performed.

Results: Total of 77 articles on DRA in postpartum women were analyzed after applying the exclusion criteria. The results were suggestive of the high global prevalence of postpartum DRA. The trend in research in the field of DRA and postpartum has increased drastically in recent years. Ultrasound imaging was found to be the most reliable tool of assessment in DRA although palpation is clinically accepted as a reliable method.

Conclusion: There is evidence from the literature supporting the high prevalence of DRA which needs to be addressed. International guidelines for the cut-off value for IRD to determine the DRA along the linea alba is lacking. Efforts are necessary to determine better strategies to prevent DRA and IRD, thereby reduce the incidence of secondary hernia and its complications. The awareness about the antenatal and postnatal care is equally important which requires contribution from clinicians across the health care profession.

Keywords: Diastasis Rectus Abdominis; Inter Rectus Distance; Postpartum; Postnatal

Abbreviatations: DRA: Diastasis Recti Abdominis; IRD: Inter Rectus Distance; LA: Linea Alba; MRI: Magnetic Resonance Imaging; CT: Computerized Tomography

\section{Introduction}

Abdominal wall is essential for the optimum functioning of the lumbo-pelvic region through multiple mechanisms which include the transfer of force through fascia by tensing it. Pregnancy affects the rectus abdominis muscle which alters its attachment due to the growing belly. Stretching and thinning of the Linea Alba (LA) increases the Inter Rectus Distance (IRD) and separates linea alba [1-4]. The alteration in the spatial relationship of muscle angle and attachment may alter the line of action of the muscle and thus their ability to produce torque [1,2,5-7]. Diastasis recti abdominis (DRA) may be defined as "an impairment characterized by the separation of the two rectus abdominis muscles along the line alba". Immediate postpartum, the prevalence of DRA above the umbilicus is $68 \%$ and that below the umbilicus is $32 \%$. DRA may result in herniation of abdominal viscera and a considerably large DRA may hamper the posture and interfere with trunk flexion, rotations, ventilarion, and trunk stability. This may cause compromised support for the organs in the abdominal and pelvic region [3,6]. A larger percentage of patients with DRA were diagnosed with urinary incontinence, fecal incontinence, pelvic organ prolapse and myofascial pelvic pain [8]. Long term manifestations of DRA are back pain, poor posture, pelvic floor problems and gastrointestinal disturbance [9].

Various methods are in practice to assess DRA, such as finger width method, ultrasonography, calipers, tape measurement, Magnetic Resonance Imaging (MRI), Computerized Tomography (CT) scans and Biodex system-4 [10,11]. Conservative management of DRA focuses on postnatal exercise which alleviates postnatal depression, limits the DRA progression, increases the general well-being of the women, improves the cardiovascular endurance and stimulates the weight loss $[12,13]$. Surgical management (Abdominoplasty) is indicated if a 
woman has failed to restore her optimal functions like transfer of load through pelvic girdle, resolve pain or pelvic floor problems and restore gastrointestinal disturbance after an optimal rehabilitation at the end of one year postpartum or if the IRD is very severe and the abdominal content can be palpated or if there is a hernia and if there is unlocking of sacroiliac joint or pubic symphysis during single leg raise $[14,15]$.

"Bibliometrics is a systemic method for evaluation of research output that can help map changes in the interest of scientific community over time and can provide insights into both quantitative and qualitative research trends on specific topic" [16]. To the best of our knowledge, there is no bibliometric analysis done specifically in this area. Hence, this study is being considered to provide an accurate survey of the published research work and examine the trends within this research discipline and also, to attempt establish the lacunae in this field of research so as to give a direction to the future research work in DRA and postpartum.

\section{Aim}

To obtain a comprehensive view of the prevalence, assessment techniques, management options and research output in the area of postpartum diastasis recti.

\section{Objectives}

a) To analyze the prevalence of DRA in postpartum women.

b) To analyze the commonly used assessment techniques for DRA in postpartum women.

c) To analyze the treatment options for the DRA in postpartum women.

d) To analyze the research output of DRA in postpartum women.

\section{Methodology}

An electronic search of Pub Med, Science Direct, Ovid, Scopus and Web of Science was performed. Key words such as 'Diastasis recti', 'Postpartum, Inter rectus distance' and 'Diastasis Recti Abdominis muscle', with boolean words 'AND/OR' was used. All the studies on the postpartum diastasis recti were included and exclusion criteria were applied. The abstracts and full articles were screened by two reviewers independently and those which fulfilled the inclusion and exclusion criteria were included. Any disagreement between the two reviewers was sorted out by a third expert. Each study was evaluated with respect to the study design, assessment techniques, intervention used, etc.

A total of 718 articles yielded by inputting the key words. Exclusion criteria were applied, based on which 627 articles were excluded as they were not relevant to this study and 14 articles were excluded as they were published in journals which were not indexed or deviated from the ethical guidelines. Seventy seven articles were selected for analysis (Figure 1). A data extraction form was structured and the analysis was performed using Microsoft excel and the data was presented in the form of counts, percentage, and frequencies.

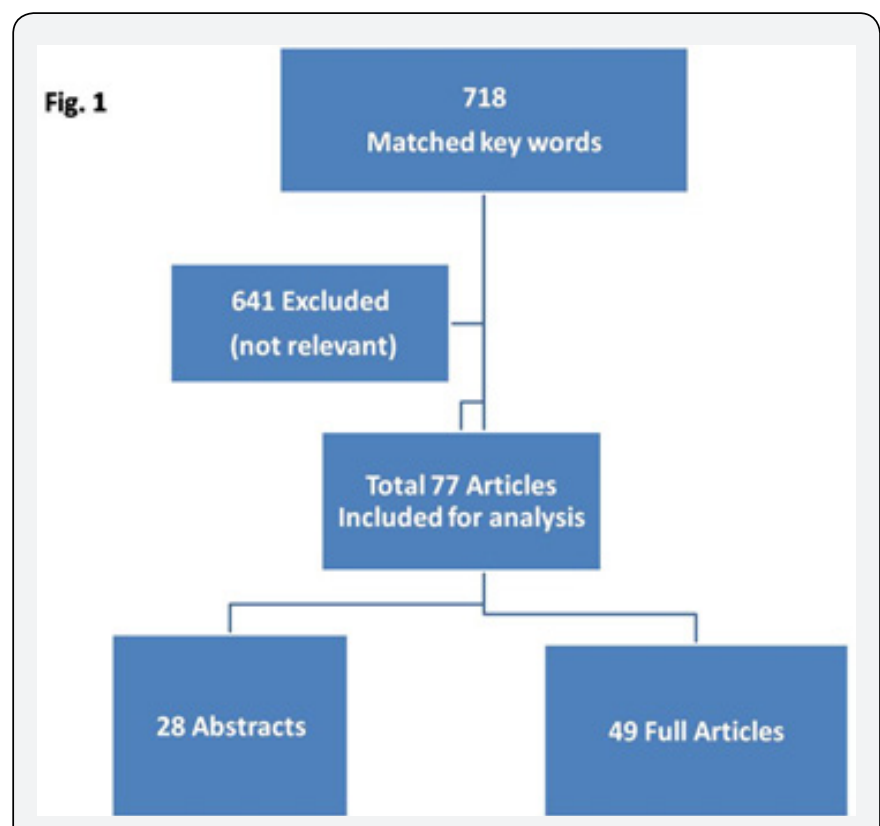

Figure 1: Flow chart depicting study selection.

Result
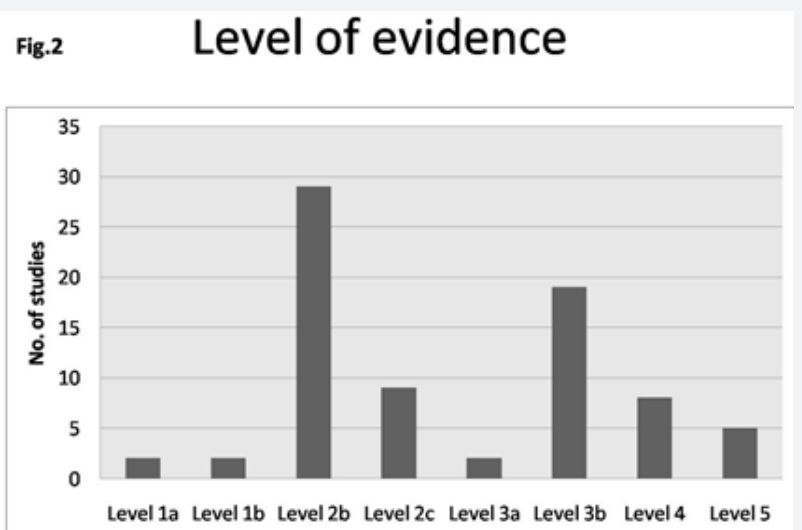

Figure 2: Column chart illustrating the types of studies under the review. The quality of study is depicted as level of evidence.

\section{Fig.3 Research Trend}

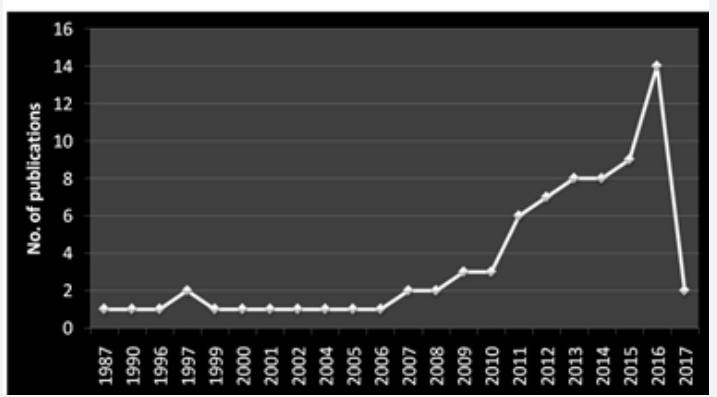

Figure 3: Line chart illustrating the number of research publications over the years. 


\section{Fig.4 Global prevalence of DRA in postpartum women}

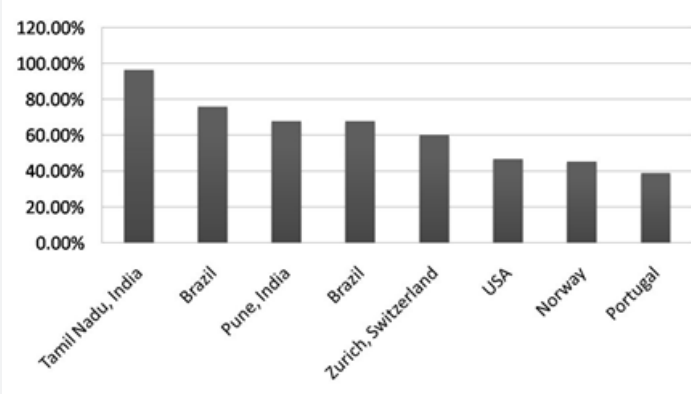

Figure 4: Column chart illustrating the global prevalence of DRA.

The study design ranged from systematic reviews and metaanalysis to case studies (Figure 2). The number of publications, contributed by over 190 authors, have increased consistently from 2011 and peaked in 2016 with 14 research publications on Diastasis recti in postpartum women (Figure 3). The prevalence of DRA in postpartum women around the globe is quite high, the highest being in India (96.55\%) (Figure 4).

\section{Fig.5 Assessment tools}

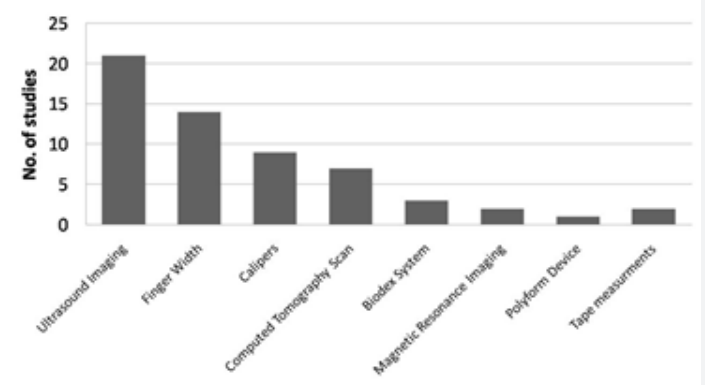

Figure 5: Column chart illustrating the commonly used tools for the assessment of DRA.

Ultrasound Imaging which is widely accepted as a reliable tool to measure DRA, was used in 22 studies. Finger width method was found to be the second most commonly used outcome measure to assess DRA and was used in 14 studies, followed by calipers, which were used in 9 studies (Figure 5).

Visual Analog Scales (Cronbach-alpha of 0.9117) [17], Pelvic Floor Distress Inventory(Cronbach's alpha 0.71-0.84) [18], Pelvic Floor Impact Questionnaire(Cronbach's alpha 0.880.94) [18] and Modified Oswestry Low Back Pain Disability Questionnaire(Cronbach's alpha 0.8107) [19] were used in the studies to rate pain, quality of life and activity of daily living outcomes. Pelvic Floor Distress Inventory(Cronbach's alpha 0.710.84) and the Pelvic Floor Impact Questionnaire (Cronbach's alpha 0.88-0.94), were used to assess low back pain and pelvic floor problems secondary to DRA.

Abdominal exercises were found to be widely used to reduce the DRA in postpartum women and were noted to yield positive results. Transverse Abdominis activation was found to be helpful along with adjuncts like Kinesio Taping and abdominal Corsets. Plastic \& Reconstructive Surgery Journal had a maximum number of citations (239) from 2001-2013 with an average impact factor of 2.10. The average impact factor of Aesthetic Plastic Surgery journal is 1.20 with 176 citations between 1997 and 2013. Journal of Orthopedic \& Sports Physical Therapy was cited 105 times between 2011and 2016 with an average impact factor of 1.80 .

\section{Discussion}

Our analysis reveals the prevalence of DRA to be $100 \%$ at gestational week and naturally fades off to around 35\% at 6 months postpartum. The findings of our study are consistent with previously published prevalence studies [1,20-22]. Furthermore the prevalence of DRA increases with parity and surgeries of the abdomen [23]. The prevalence of DRA was similar among multipara and primipara at umbilicus but below the umbilicus, multipara had more [6]. DRA is usually linked to many other conditions like myofascial pelvic pain 33\%, urinary incontinence $48 \%$, fecal incontinence $7 \%$, uterus prolapse $52 \%$, bladder prolapse $57 \%$ and rectal prolapse $43 \%$. Studies have shown an association of DRA with conditions like low back pain and lumbo-pelvic pain [21,23-25].

According to 'Noble Criteria', DRA is said to be positive if a gap of 2 finger width is present at the umbilicus, above the umbilicus or below [26]. One of the studies that was published in the year 1987 states that the anterior aspect of the rectus sheath is presumed to be stronger below the umbilicus as all the four muscles of anterior abdominal wall cross below the umbilicus, thus the additional reinforcement prevent the separation of Rectus Abdominis below umbilicus. The two heads of the rectus abdominis muscle resemble "V" as they originate at the pubis. The criteria of more than two finger widths, therefore, may not be appropriate below the umbilicus. A separation of greater than one finger width might be indicative of significant DRA below the umbilicus [27]. A cadaver study carried out in 1996 concludes that IRD more than $10 \mathrm{~mm}$ above the umbilicus, $27 \mathrm{~mm}$ at the level of the umbilicus and $9 \mathrm{~mm}$ below the umbilicus could be pathological DRA [28]. Another study published in 2005 proclaims DRA as a widening of the IRD more than $2.5 \mathrm{~cm}$ at one or more assessment points using digital calipers [13]. A study published in 2009 suggests that in nulliparous women, the linea alba should be considered normal when the IRD width is less than $15 \mathrm{~mm}$, at the xiphoid level, $22 \mathrm{~mm}$ at $3 \mathrm{~cm}$ above the umbilicus and $16 \mathrm{~mm}$ at a level $2 \mathrm{~cm}$ below the umbilicus [29]. Hence, standardization in this regard needs to be established.

Taking in to consideration the findings of the published research works analyzed in the current study, we could conclude that ultrasound scanning is the gold standard tool to assess DRA and is the most widely used tool for this purpose and is also valid and highly reliable [29-32]. Ultrasound imaging has a limitation when used on larger IRD, which is substantiated 
by a study carried out in 2015. A study in 2013 was conducted to check the concurrent validity of digital nylon calipers and ultra sound imaging (USI) with respect to IRD measurement. For the measurements of IRD with calipers, ICC of 0.79 and 0.71 with abdominal muscles at rest and abdominal muscles contracted respectively were documented, which were similar to measurements made with USI above the umbilicus. The values obtained below the umbilicus with caliper and USI were not comparable [33].

Clinically finger width method and tape measurements have been used, probably because of its cost effectiveness and accessibility. Palpation showed good intra-rater reliability between days which was expressed by a weighted Kappa (wK) higher than 0.7 for two assessors and moderate inter-rater reliability (wK $1 / 40.534$ ). This may be attributed to variations in the assessors' experience, finger width and soft tissue pressure. A study in 2012 on test-retest and intra-rater reliability of palpation recorded an intra-class correlation coefficient (ICC) value ranging between 0.74 and 0.90 for test-retest measurements. For the intra-rater reliability, ICC values were above 0.90 [34]. Palpation has enough reliability to be used in the clinical setup but USI is highly accurate. Till date, there are no published studies available to assess the reliability of tape measurements.

Even though the prevalence of DRA is high, there are only a few intervention studies which gives a clear picture as to which exercises are beneficial for preventing and managing DRA. Antenatal exercise has also proved to be beneficial in the prevention and management of DRA. A study in 1999 states that 'drawing-in' helps in reducing DRA [35] but a couple of studies $[20,36,37]$. contradict the statement by stating 'drawing-in' resulted in the widening of the IRD. It is worth noting that these were one time studies aiming to check the effect of 'drawing-in' and not interventional and lacked long term follow up. In 2005 a study demonstrated that the prevalence of DRA was less when the women did antenatal exercises compared to women who did not exercise [13]. There is a need for the development and conduct of standardized interventions for the prevention and management of DRA.

\section{Conclusion}

There is evidence from the literature clearly supporting the high prevalence of DRA even after 6 months postpartum which needs to be addressed. There is need for international guidelines for the cut-off value for IRD to determine the DRA along the linea alba. There is a need for a standardized Questionnaire for DRA and its validation which may be universally accepted. The literature review reveals that till date only one published randomized control trial exists pertaining to exercise and kinesio taping in patients with postpartum DRA. Therefore, there is a clear need for high-quality research to determine the best strategy to prevent DRA and reduce the IRD, in an attempt to minimize the associated consequences. It may be recommended that pregnant women be educated about the DRA and its consequences so as to prevent it right from the antenatal period. The awareness about the antenatal and postnatal care is equally important.

\section{Limitation}

There have been best possible efforts made to extract the research work from the search engines but the possibilities of manual error may not be ruled out. Efforts have been made to translate the non- English publications within our limitations. Since the ongoing trials were excluded, we cannot, therefore, avoid the possibility that we may not have included some eligible studies.

\section{References}

1. Hsia M, Jones S (2000) Natural resolution of rectus abdominis diastasis. Two single case studies. Aust J Physiother 46(4): 301-307.

2. Lee D (2016) Behavior of the Linea Alba During a Curl-up Task in Diastasis Rectus Abdominis: An Observational Study. J Orthop Sports Phys Ther 46(7): 580-589.

3. Misha A, Maria L, Umar M, Raheela K (2014) Prevalence of Diastasis Recti among Pregnant Females Presenting at Holy Family Hospital Rawalpindi. IJRS pp. 26-31.

4. Litos K (2014) Progressive Therapeutic Exercise Program for Successful Treatment of a Postpartum Woman with a Severe Diastasis Recti Abdominis. Journal of Women's Health Physical Therapy 38(2): 58-73.

5. Gilleard WL (1996) Structure and function of the abdominal muscles in primigravida during pregnancy and the immediate post-birth period Structure and Function of the Abdominal Muscles in Prirnigravid Subjects During Pregnancy and the Immediate Postbirth Period. Phys Ther 76(7): 750-762.

6. Rett M, Braga M, Bernardes N, Andrade S (2009) Prevalence of diastasis of the rectus abdominis muscles immediately postpartum : comparison between primiparae and multiparae. Revista Brasileira de Fisioterapia 13(4): 275-280.

7. Mota PGFda, Pascoal AG, Carita AI, Bo K (2015) Prevalence and risk factors of diastasis recti abdominis from late pregnancy to 6 months postpartum, and relationship with lumbo-pelvic pain. Man Ther 20(1): 200-205.

8. Spitznagle TM, Leong FC (2007) The Relationship Between Diastasis Recti Abdominis and Pelvic Floor Dysfunction Diagnoses. Journal of Women's Health Physical Therapy 31(1): 1-2.

9. Mantle J, Jeanette H, Sue B (1978) Physiotherapy in obsteratics and gynaecology. Br Med J 2(6150): 1497.

10. van de Water AT, Benjamin DR (2016) Measurement methods to assess diastasis of the rectus abdominis muscle ( DRAM ): A systematic review of their measurement properties and meta-analytic reliability generalisation. Man Ther 21: 41-53.

11. Stark B, Emanuelsson P, Gunnarsson U, Strigård K (2012) Validation of Biodex system 4 for measuring the strength of muscles in patients with rectus diastasis. J Plast Surg Hand Surg 46(2): 102-105.

12. Gürşen C, İnanoğlu D, Kaya S, Akbayrak T, Baltacı G (2015) Effects of exercise and Kinesio taping on abdominal recovery in women with cesarean section: a pilot randomized controlled trial. Arch Gynecol Obstet 293(3): 557-565.

13. Chiarello CM, Falzone La, McCaslin KE, Patel MN, Ulery KR, et al. (2005) The Effects of an Exercise Program on Diastasis Recti Abdominis in Pregnant Women. Journal of Women's Health Physical Therapy, 29(1): 11-16. 
14. Lee DG, Lee LJ, McLaughlin L (2008) Stability, continence and breathing: The role of fascia following pregnancy and delivery. J Bodyw Mov Ther 12(4): 333-348.

15. Hickey F, Finch JG, Khanna A (2011) A systematic review on the outcomes of correction of diastasis of the recti. Hernia 15(6): 607-614.

16. Ugolini D, Neri M, Cesario A, Marazzi, G, Milazzo D, et al. (2013) Bibliometric Analysis of Literature in Cerebrovascular and Cardiovascular Diseases Rehabilitation: Growing Numbers, Reducing Impact Factor Arch Phys Med Rehabil 94(2): 324-331.

17. Knop C, Oeser M, Bastian L, Lange U, Zdichavsky M, et al. (2001) Development and validation of the Visual Analogue Scale (VAS) Spine Score. Der Unfallchirurg 104(6): 488-497.

18. Sultan AH, Monga A, Lee J, Emmanuel A, Norton C, et al. (2017) An International Urogynecological Association (IUGA)/International Continence Society (ICS) joint report on the terminology for female anorectal dysfunction. Int Urogynecol J 28(1): 5-31.

19. Fairbank JC, Couper J, Davies JB, O'Brien JP (1980) The Oswestry low back pain disability questionnaire. Physiotherapy 66(8): 271-273.

20. Sancho MF, Pascoal AG, Mota P, Bø K (2015) Abdominal exercises affect inter-rectus distance in postpartum women: a two-dimensional ultrasound study. Physiotherapy 101(3): 286-291.

21. Gitta S, Magyar Z, Tardi P, Füge I, Járomi M, et al. (2017) Prevalence, Potential Risk Factors and Sequelae of Diastasis Recti Abdominis. Orvosi Hetilap 158(12): 454-460.

22. Ashwini SK, Shital NG, Bhave SM, NK D (2017) A survey on prevalence of diastasis of the rectus abdominis muscles in puerperium: primiparous and multiparous and in second trimester and immediate postpartum period, pp. 1-2.

23. Turan V, Colluoglu C, Turkyilmaz E, Korucuoglu U (2011) Prevalence of diastasis recti abdominis in the population of young multiparous adults in Turkey. Ginekol Pol 82(11): 817-821.

24. Spitznagle TM, Leong FC, Van Dillen LR (2007) Prevalence of diastasis recti abdominis in a urogynecological patient population. Int Urogynecol J Pelvic Floor Dysfunct 18(3): 321-328.

25. Khushboo D, Amrit Kaur MM (2014) Correlation between Diastasis Rectus Abdominis and Lumbopelvic Pain and Dysfunction. Indian Journal of Physiotherapy \& Occupational Therapy-An International Journal 8(1): 210-214.
26. E N (1982) Essential Exercises for the Childbearing Year. In: ( $\left.2^{\text {nd }} e d n\right)$.

27. Blaschak MJO (2013) Incidence of Diastasis Recti Abdominis During the Childbearing Year.

28. Beer GM, Schuster A, Seifert B, Manestar M, Mihic-Probst D, et al. (2009) The normal width of the linea alba in nulliparous women. Clinical Anatomy 22(6): 706-711.

29. Keshwani N, Hills N, McLean L (2016) Inter-Rectus Distance Measurement Using Ultrasound Imaging: Does the Rater Matter? Physiother Can 68(3): 223-229.

30. Keshwani N, Mathur S, McLean L (2015) Validity of Inter-rectus Distance Measurement in Postpartum Women Using Extended Field of View ultrasound Imaging Techniques. J Orthop Sports Phys Ther 45(10): 808-813.

31. Keshwani N, McLean L (2015) Ultrasound Imaging in Postpartum Women with Diastasis Recti: Intrarater Between-Session Reliability. J Orthop Sports Phys Ther 45(9): 713-718.

32. Iwan T, Physiotherapy B, Garton B, Ellis R (2013) The reliability of measuring the inter-recti distance using high-resolution and low-resolution ultrasound imaging comparing a novice to an experienced sonographer. Ml Roberts Prize Winner 42(2): 154-163.

33. Chiarello CM, McAuley JA (2013) Concurrent Validity of Calipers and Ultrasound Imaging to Measure Interrecti Distance. J Orthop Sports Phys Ther 43(7): 495-503.

34. Mota P, Ag P, Sancho F, Bø K (2012) Test-retest and intrarater reliability of 2-dimensional ultrasound measurements of distance between rectus abdominis in women. J Orthop Sports Phys Ther 42(11): 940-946.

35. Mesquita LA, Machado AV (1999) Physical Therapy to Reduce Muscle Dysthesis Abdominal Challenges in the Postpartum Physiotherapy for Reduction of Diastasis of the Recti Abdominis Muscles in the Postpartum Period, pp. 1-9.

36. Mota P, Pascoal AG, Carita AI, Bø K (2015) The Immediate Effects on Inter-rectus Distance of Abdominal Crunch and Drawing-in Exercises During Pregnancy and the Postpartum Period. J Orthop Sports Phys Ther 45(10): 781-788.

37. Mota P, Pascoal AG, Carita AI, Bø K (2015) Inter-recti distance at rest, during abdominal crunch and drawing in exercises during pregnancy and postpartum. Physiotherapy 101(1): e1050-e1051.

\section{Your next submission with Juniper Publishers will reach you the below assets}

- Quality Editorial service

- Swift Peer Review

- Reprints availability

- E-prints Service

- Manuscript Podcast for convenient understanding

- Global attainment for your research

- Manuscript accessibility in different formats

( Pdf, E-pub, Full Text, Audio)

- Unceasing customer service

Track the below URL for one-step submission

https://juniperpublishers.com/online-submission.php 